\title{
Identification of Genes and Pathways of Nonsteroidal Anti-Inflammatory Drugs Acting on Synovia from Women with Knee Osteoarthritis by Bioinformatics Analysis
}

Chao Zhao ( $\nabla$ chaozhao0311@163.com )

Third Hospital of Hebei Medical University https://orcid.org/0000-0002-4030-6384

Han Wang

Second Hospital of Hebei Medical University

Conglei Dong

Third Hospital of Hebei Medical University

Huijun Kang

Third Hospital of Hebei Medical University

Fei Wang

Third Hospital of Hebei Medical University

\section{Research Article}

Keywords: nonsteroidal anti-inflammatory drugs, knee osteoarthritis, synovia, bioinformatics analysis

Posted Date: August 2nd, 2021

DOI: https://doi.org/10.21203/rs.3.rs-407139/v2

License: (c) (1) This work is licensed under a Creative Commons Attribution 4.0 International License.

Read Full License 


\section{Abstract}

Purpose: Through the bioinformatics analysis, to identify the genes and pathways of nonsteroidal antiinflammatory drugs(NSAIDs) acting on synovia from women with knee osteoarthritis (KOA), and to provide reference for clinical application.

Methods: We downloaded the gene microarray datasets with the accession number of GSE55457 and GSE55584 from the Gene Expression Omnibus (GEO; https://www.ncbi.nlm.nih.gov/geo/) database, including 5 untreated KOA patients, 9 NSAIDs treated KOA patients and 2 patients without KOA The samples in the untreated KOA group and the NSAIDs treated KOA group were used for main analysis. The samples in the untreated KOA group and the normal control group were used for cooperative analysis. Then we performed robust multi-array (RMA) normalization with affy R programming package. After that, differential expression genes (DEGs) in main analysis and cooperative analysis were identified based on limma package separately. Screening the common DEGs from main analysis and cooperative analysis. Enriched gene ontology (GO) terms and Kyoto Encyclopedia of Genes and Genomes (KEGG) pathways of DEGs were obtained through the Database for Annotation, Visualization and Integrated Discovery (DAVID). What's more, protein-protein interaction (PPI) network was constructed, and we identified modules of PPI network through Cytoscape to screen valuable targets. The value of gene expression fold change $(F C) \geq 1.4$ or $\leq 1 / 1.4$, and $P<0.05$ were used as the screening conditions. $P<0.05$ and Associated genes count $>5$ were used as the screening conditions.

Results: There were 338 DEGs in main analysis. Among them, 211 genes were up-regulated and 127 genes were down-regulated. There were 7005 DEGs in cooperative analysis. Among them, 6952 genes were up-regulated and 53 genes were down-regulated. A total of 129 common DEGs were identified between main analysis and cooperative analysis. There are 2 biological processes, 3 cell components and 2 molecular functions for the enrichment of differentially expressed genes.

Conclusion: NSAIDs may play a certain role in synovia from women with KOA by regulating the mRNA expressions of il-6, TNFRSF11A and CSF1R, which may become one of the indicators for monitoring the efficacy of NSAIDs.

\section{Introduction}

Knee osteoarthritis (KOA) is a degenerative disease characterized by degeneration of articular cartilage (1), which currently affects about $21.51 \%$ of the middle-aged and elderly (2), and is considered as one of the largest cause of disability (3). The genetic contribution of knee osteoarthritis surgery is higher in women than in men (4). KOA is often accompanied by synovitis, osteophyte formation and subchondral bone sclerosis (5). Clearly, all articular tissues, including the synovium, are involved in the overall pathologic process. In particular, synovitis, which plays a key role in the initiation and development of degenerative changes in cartilage, is associated with more severe pain and joint dysfunction (6). Synoviocytes produce pro-inflammatory mediators, which in turn attract immune cells, increase 
angiogenesis and induce a phenotypic shift in chondrocytes. Chondrocytes produce additional cytokines and proteolytic enzymes, which eventually increase cartilage degradation and further induce synovial inflammation (7). Therefore, the study on synovial tissue of KOA patients will gradually attract extensive attention.

Nonsteroidal anti-inflammatory drugs (NSAIDs) are a class of widely used drugs with antipyretic, analgesic and anti-inflammatory effects and it is often used in the clinical treatment of KOA, rheumatoid arthritis and other inflammatory diseases (8). It mainly inhibits the release and production of prostaglandin synthetase (PGs) by inhibiting the activity of cox-oxidase (COX) and other pathways, thus exerting the anti-inflammatory effect (9). In addition, NSAIDs can also adjust the function of osteoblasts and osteoclasts by inhibiting PGs through the above pathways (10). However, there is little evidence that they can alter or stop the progression of KOA (11). Due to its general need for long-term oral, the resulting toxic side effects such as gastrointestinal reactions, cardiovascular side effects can not be ignored (12, 13). Therefore, the indications for its application are open to question, and many mechanisms are unknown. NSAIDs last long in synovial fluid, and considerable concentrations of drugs can be achieved in synovial fluid (14). However, little research has been done on whether NSAIDs play an intervention role in the expression of abnormal genes in synovial tissues of KOA patients and the details of its mechanism. Through the bioinformatics analysis, we tried to identify the genes and pathways of NSAIDs acting on synovia from women with KOA. We downloaded the gene microarray datasets with the accession number of GSE55457 and GSE55584 from the Gene Expression Omnibus (GEO; https://www.ncbi.nlm.nih.gov/geo/) database, performed robust multi-array (RMA) normalization with affy $R$ programming package, and differential expression genes (DEGs) in two groups were identified based on limma package separately for main analysis and cooperative analysis (15). Through our further analysis, to provide reference for clinical application.

\section{Materials And Methods}

\section{Microarray data.}

We downloaded the gene microarray datasets with the accession number of GSE55457 and GSE55584 from the GEO database, including 14 samples of synovia from women with KOA and 2 samples of synovia from women without KOA. Among them, there were $5 \mathrm{KOA}$ patients in the untreated KOA group (untreated KOA) (GSM1337327, GSM1337330 from GSE55457 dataset, and GSM1339628, GSM1339629, GSM1339632 from GSE55584 dataset), 9 KOA patients in the NSAIDs treated KOA group (NSAIDs treated KOA) (GSM1337328, GSM1337329, GSM1337331, GSM1337334, GSM1337335, GSM1337336 from GSE55457 dataset, and GSM1339630, GSM1339631, GSM1339633 from GSE55584 dataset), and 2 patients without KOA in the normal control group (normal control) (GSM1337306 and GSM1337310 from GSE55457 dataset).The samples in the untreated KOA group and the NSAIDs treated KOA group were used for main analysis. To repeat the grouping of BOBIN MI,the samples in the untreated KOA group and the normal control group were used for cooperative analysis. 


\section{Identification of DEGs.}

To identify DEGs for main analysis and cooperative analysis, we did the following several times. We performed RMA normalization with affy R programming package. After that, DEGs in two groups were identified based on limma package separately for main analysis and cooperative analysis. The screening criteria for DEGs were: the value of gene expression fold change $(F C) \geq 1.4$ or $\leq 1 / 1.4$, and $P<0.05$.

\section{Screening for common DEGs.}

Screening the common differentially expressed genes (DEGs) from main analysis and cooperative analysis.

\section{Gene ontology(GO) and Kyoto Encyclopedia of Genes and Genomes (KEGG) pathway enrichment analysis for common DEGs.}

Enriched GO terms and KEGG pathways of common DEGs were obtained through the Database for Annotation, Visualization and Integrated Discovery (DAVID). For screening results, $\mathrm{P}<0.05$ and Associated genes count $>5$ were used as the screening conditions.

\section{PPI network analysis for common DEGs.}

PPI network was constructed by using the web-based tool STRING (http://www.string-db.org). Subsequently, the PPI network was visualized by using Cytoscape software (http://www.cytoscape.org/).

\section{Results}

\section{Identification of DEGs for main analysis}

The untreated KOA group and the NSAIDs treated KOA group had good clustering and no outlier samples, and all the 14 samples could be used for further analysis. $F C \geq 1.4$ or $\leq 1 / 1.4$, and $P<0.05$ were used as the screening conditions. There were $338 \mathrm{DEGs}$ in total between the untreated KOA group and the NSAIDs treated KOA group. Among them, 211 genes were up-regulated and 127 genes were down-regulated. (Table 1 and Table 2). 
Table 1

Upregulated top 10 DEGs for main analysis

\begin{tabular}{|c|c|c|}
\hline Name & ad.j P value & $\log _{2} \mathrm{FC}$ \\
\hline ERAP2 & 0.039802988 & 2.204622561 \\
\hline EGFL6 & 0.010200799 & 1.639875579 \\
\hline CLEC5A & 0.000370042 & 1.357457197 \\
\hline HYPM & 1.18E-06 & 0.978032929 \\
\hline GPC5 & 0.046163148 & 0.975027997 \\
\hline METTL8 & 8.11E-07 & 0.89670674 \\
\hline METTL2B & 0.000132549 & 0.883810499 \\
\hline RBM41 & 1.96E-05 & 0.866861208 \\
\hline RSBN1 & 0.000589657 & 0.863816353 \\
\hline KIAA1462 & 0.015328306 & 0.860426184 \\
\hline \multicolumn{3}{|c|}{$\begin{array}{l}\text { DEGs: differential expression genes; ERAP2: endoplasmic reticulum aminopeptidase 2; EGFL6: } \\
\text { epidermal growth factor like domain multiple 6; CLEC5A: C-type lectin domain containing 5A; HYPM: } \\
\text { huntingtin interacting protein M; GPC5: glypican 5; METTL8: methyltransferase like 8; METTL2B: } \\
\text { methyltransferase like 2B; RBM41: RNA binding motif protein 41; RSBN1: round spermatid basic } \\
\text { protein 1; KIAA1462: Junctional Protein Associated with Coronary Artery Disease. }\end{array}$} \\
\hline
\end{tabular}


Table 2

Down-regulated top 10 DEGs for main analysis

\begin{tabular}{|c|c|c|}
\hline Name & ad.j P value & $\log _{2} F C$ \\
\hline LOC100293211 & 0.008071821 & -1.999464744 \\
\hline TNNC1 & 0.047915938 & -1.936767919 \\
\hline IGK & 0.03001768 & -1.835423764 \\
\hline ACPP & $1.06 \mathrm{E}-07$ & -1.738148309 \\
\hline TNNT3 & 2.75E-05 & -1.658828783 \\
\hline STEAP4 & 0.017920088 & -1.625012329 \\
\hline TNKS2 & 0.00318571 & -1.622724424 \\
\hline IGLV1-44 & 0.020105589 & -1.610300515 \\
\hline AMPD1 & 0.003600788 & -1.583795204 \\
\hline PCSK1 & 0.01179745 & -1.562040655 \\
\hline \multicolumn{3}{|c|}{$\begin{array}{l}\text { DEGs: differential expression genes; LOC100293211: This record has been withdrawn by NCBI } \\
\text { because the model on which it was based was not predicted in a later annotation; TNNC1: troponin } \\
\text { C1, slow skeletal and cardiac type; IGK: immunoglobulin kappa locus; ACPP: acid phosphatase, } \\
\text { prostate; TNNT3: troponin T3, fast skeletal type; STEAP4: six transmembrane epithelial antigen of the } \\
\text { prostate 4; TNKS2: tankyrase 2; IGLV1-44: immunoglobulin lambda variable 1-44; AMPD1: adenosine } \\
\text { monophosphate deaminase 1; PCSK1: proprotein convertase subtilisin/kexin type 1. }\end{array}$} \\
\hline
\end{tabular}

\section{Identification of DEGs for cooperative analysis.}

The untreated KOA group and the normal control group had good clustering and no outlier samples, and all the 7 samples could be used for further analysis. $\mathrm{FC} \geq 1.4$ or $\leq 1 / 1.4$,and $\mathrm{P}<0.05$ were used as the screening conditions. There were 7005 DEGs in total between the normal control group and untreated KOA. Among them, 6952 genes were up-regulated and 53 genes were down-regulated. (Table 3 and Table 4). 
Table 3

Upregulated top 10 DEGs for cooperative analysis

\begin{tabular}{|lll|}
\hline Name & ad.j P value & $\log _{2} \mathrm{FC}$ \\
\hline CSN1S1 & 0.002915198 & 3.753268436 \\
\hline SCRG1 & 0.004842648 & 3.393259056 \\
\hline IGJ & 0.037337558 & 2.98865458 \\
\hline CXCL13 & 0.029616323 & 2.951166838 \\
\hline FCER1A & 0.020646921 & 2.911387621 \\
\hline LOC100293211 & 0.033474633 & 2.780834072 \\
\hline SFRP4 & 0.013146367 & 2.652018346 \\
\hline CXCL14 & 0.003753975 & 2.642478092 \\
\hline CPA3 & 0.003248469 & 2.53155903 \\
\hline $\begin{array}{l}\text { AMPD1 } \\
\text { DEGs: differential expression genes; CSN1S1: casein alpha s1; SCRG1: stimulator of chondrogenesis }\end{array}$ \\
$\begin{array}{l}\text { 1; IGJ: joining chain of multimeric lgA and IgM; CXCL13: C-X-C motif chemokine ligand 13; FCER1A: } \\
\text { FC fragment of IgE receptor la; LOC100293211: This record has been withdrawn by NCBI because the } \\
\text { model on which it was based was not predicted in a later annotation; SFRP4: secreted frizzled related } \\
\text { protein 4; CXCL14: C-X-C motif chemokine ligand 14; CPA3: carboxypeptidase A3; AMPD1: adenosine } \\
\text { monophosphate deaminase 1. }\end{array}$ \\
\hline
\end{tabular}


Table 4

Down-regulated top $10 \mathrm{DEGs}$ for cooperative analysis

\begin{tabular}{|lll|}
\hline NameName & ad.j P value & $\log _{2} \mathrm{FC}$ \\
\hline DIP2A & 0.003753975 & -1.209337988 \\
\hline KATNBL1 & 0.006498532 & -1.11132739 \\
\hline ANKRD36B & 0.002108899 & -1.083693789 \\
\hline RUFY2 & 0.027166392 & -1.058688078 \\
\hline ADH1A & 0.014392153 & -1.053951323 \\
\hline WDR43 & 0.003117186 & -1.044556176 \\
\hline HYMAI & 0.016933768 & -1.030648055 \\
\hline CD2AP & 0.00500248 & -1.029287464 \\
\hline SCN4A & 0.046500125 & -1.02868922 \\
\hline $\begin{array}{l}\text { ACTL6A } \\
\text { DEGs: differential expression genes; DIP2A: disco interacting protein 2 homolog A; KATNBL1: katanin } \\
\text { regulatory subunit B1 like 1; ANKRD36B: ankyrin repeat domain 36B; RUFY2: RUN and FYVE domain } \\
\text { Containing 2; ADH1A: alcohol dehydrogenase 1A (class I), alpha polypeptide; WDR43: WD repeat } \\
\text { domain 43; HYMAl: hydatidiform mole associated and imprinted; CD2AP: CD2 associated protein; } \\
\text { SCN4A: sodium voltage-gated channel alpha subunit 4; ACTL6A: actin like 6A. }\end{array}$ \\
\hline
\end{tabular}

\section{Screening for common DEGs.}

Screening the common DEGs from main analysis and cooperative analysis. There were 129 common DEGs in total between main analysis and cooperative analysis. (Fig. 1).

\section{GO and pathway enrichment analysis for common DEGs.}

For screening results, $\mathrm{P}<0.05$ and Associated genes count $>5$ were used as the screening conditions. There were 7 results that have been filtered out. Among them, there are 2 biological processes(BP), 3 cell components(CC) and 2 molecular functions(MF) for the enrichment of differentially expressed genes. (Table 5). There was 0 KEGG pathway enriched by differentially expressed genes. 
Table 5

The result of Enriched GO terms and KEGG pathways of common DEGs

\begin{tabular}{|lllll|}
\hline Term & ID & Function & $\begin{array}{l}\text { Associated genes } \\
\text { count }\end{array}$ & P Value \\
\hline BP & G0:0008284 & positive regulation of cell proliferation & 9 & 0.012782 \\
BP & G0:0043066 & $\begin{array}{l}\text { negative regulation of apoptotic } \\
\text { process }\end{array}$ & 8 & 0.032684 \\
\hline CC & G0:0009986 & cell surface & 15 & $1.33 \mathrm{E}-05$ \\
\hline CC & GO:0005887 & $\begin{array}{l}\text { integral component of plasma } \\
\text { membrane }\end{array}$ & 20 & 0.002222 \\
\hline CC & GO:0005886 & plasma membrane & 0.036567 \\
\hline MF & GO:0019901 & protein kinase binding & 8 & 0.012663 \\
\hline MF & GO:0005524 & ATP binding & 17 & 0.037856 \\
\hline $\begin{array}{l}\text { GO: gene ontology; KEGG: Kyoto Encyclopedia of Genes and Genomes; BP: biological processes; CC: } \\
\text { cell components; MF: } \text { molecular functions; }\end{array}$ & & \\
\hline
\end{tabular}

\section{PPI network analysis for common DEGs.}

Based on the information in the STRING and Cytoscape databases, the top 10 hub nodes with the highest degree of interaction were screened: CD24, CD70, KCNC1, BIRC3, TNFRSF11A, CSF1R, HP, CD69, CD40LG, and IL6. (Fig. 2).

\section{Discussion}

In this study, the discussion of DEGs, related pathways and PPIs in synovial tissue of female KOA patients after NSAIDs treatment is helpful to strengthen the understanding of researchers on KOA and NSAIDs. In this study, CD24, CD70, KCNC1, BIRC3, TNFRSF11A, CSF1R, HP, CD69, CD40LG, and IL6 may play an important role in the synovial tissue of female KOA patients after NSAIDs treatment. In addition, NSAIDs treatment will also affect a series of pathways: "positive regulation of cell proliferation", "negative regulation of apoptotic process", "cell surface", "integral component of plasma membrane", "plasma membrane", and "protein kinase Binding", "ATP binding". IL-6 is a soluble mediator that has multipotency effects on inflammation, immune response and hematopoiesis (16). It can not only stimulate the aggregation and activation of inflammatory cells, but also promote the formation of pain of the knee joint after trauma (17). IL-6 can mediate cartilage destruction (18). By stimulating synovial cells, IL-6 can produce PGs, which further aggravates joint inflammation, activates immature osteoclasts, and makes them participate in bone resorptive, causing progressive destruction of articular cartilage $(18,19)$. 
TNFRSF11A is a member of the tumor necrosis factor receptor superfamily, which interacts with a variety of TRAF family proteins to induce the activation of NF-Kappa B and MAPK signaling pathway, and is also an important mediator of osteoclast development (20-23). CSF1 can stimulate CSF1R to promote the proliferation and differentiation of macrophages and the formation of osteoclasts, so in patients with KOA, inhibition of CSF1R is beneficial (24-26).

The limitation of this study is that only a small number of synovium samples were included, and the inclusion of other samples may change the current results. Therefore, it is necessary to collect more synovial samples from female patients with KOA to detect the expression levels of significant DEGs. In addition, the significance of PTGS1 and PTGS2 changes was relatively low, which was surprising.

In conclusion, NSAIDs may play a certain role in synovium of female KOA by regulating the mRNA expression of IL-6, TNFRSF11A and CSF1R, which may become one of the indicators for monitoring the efficacy of NSAIDs. Given their reported side effects, they should be appropriately recommended.

\section{Abbreviations}

NSAIDs: nonsteroidal anti-inflammatory drugs;

KOA: knee osteoarthritis;

GEO: Gene Expression Omnibus;

RMA: robust multi-array;

DEGs: differential expression genes;

GO: gene ontology;

KEGG: Kyoto Encyclopedia of Genes and Genomes;

DAVID: Database for Annotation, Visualization and Integrated Discovery;

PPI: protein-protein interaction;

FC: fold change;

PGs: prostaglandin synthetase;

COX: cox-oxidase;

BP: biological processes;

CC: cell components;

MF: molecular functions; 


\section{Declarations}

\section{Acknowledgements}

Thanks to Woetzel $D$ et al for the raw data published in the open database.

\section{Author's Contributions}

Fei Wang contributed to the conception of the study; Chao Zhao contributed significantly to the analysis and wrote the manuscript; Han Wang collected the data; Conglei Dong and Huijun Kang helped perform the analysis with constructive discussions. The authors read and approved the final manuscript.

\section{Funding}

Funded by The National Natural Science Foundation of China (Grant No.81873983)

\section{Availability of data and materials}

All of the data and materials are available online.

\section{Ethics approval and consent to participate}

The present study was approved by the Academic Ethics Committee of the Third Hospital of Hebei Medical University.

\section{Consent for publication}

Not applicable.

\section{Competing interests}

The authors declare that they have no competing interests.

\section{References}

1. Oláh T, Reinhard J, Gao L, et al.: Topographic modeling of early human osteoarthritis in sheep. Sci Transl Med 112019.

2. Sun X, Zhen X, Hu X, et al.: Osteoarthritis in the Middle-Aged and Elderly in China: Prevalence and Influencing Factors. Int J Environ Res Public Health 162019. 
3. Hunter DJ, Bierma-Zeinstra S. Osteoarthritis Lancet. 2019;393:1745-59.

4. Magnusson K, Turkiewicz A, Englund M. Nature vs nurture in knee osteoarthritis - the importance of age, sex and body mass index. Osteoarthritis Cartilage. 2019;27:586-92.

5. Charlier E, Deroyer C, Ciregia F, et al. Chondrocyte dedifferentiation and osteoarthritis (OA). Biochem Pharmacol. 2019;165:49-65.

6. Scanzello CR, Goldring SR. The role of synovitis in osteoarthritis pathogenesis. Bone. 2012;51:24957.

7. Mathiessen A, Conaghan PG. Synovitis in osteoarthritis: current understanding with therapeutic implications. Arthritis Res Ther. 2017;19:18.

8. Braun J, Baraliakos X, Westhoff T. Nonsteroidal anti-inflammatory drugs and cardiovascular risk - a matter of indication. Semin Arthritis Rheum. 2020;50:285-8.

9. Goldenberg MM. Celecoxib, a selective cyclooxygenase-2 inhibitor for the treatment of rheumatoid arthritis and osteoarthritis. Clin Ther. 1999;21:1497-513. discussion 1427-1498.

10. Kellinsalmi M, Parikka V, Risteli J, et al. Inhibition of cyclooxygenase-2 down-regulates osteoclast and osteoblast differentiation and favours adipocyte formation in vitro. Eur J Pharmacol. 2007;572:102-10.

11. Derry S, Conaghan P, Da Silva JA, Wiffen PJ, Moore RA: Topical NSAIDs for chronic musculoskeletal pain in adults. The Cochrane database of systematic reviews 4: Cd007400, 2016.

12. Um SY, Chung MW, Kim KB, et al. Pattern recognition analysis for the prediction of adverse effects by nonsteroidal anti-inflammatory drugs using $1 \mathrm{H}$ NMR-based metabolomics in rats. Anal Chem. 2009;81:4734-41.

13. Ricciotti E, Castro C, Tang SY, et al. Cyclooxygenase-2, Asymmetric Dimethylarginine, and the Cardiovascular Hazard From Nonsteroidal Anti-Inflammatory Drugs. Circulation. 2018;138:2367-78.

14. Davies NM. Clinical pharmacokinetics of ibuprofen. The first 30 years. Clin Pharmacokinet. 1998;34:101-54.

15. Woetzel D, Huber R, Kupfer P, et al. Identification of rheumatoid arthritis and osteoarthritis patients by transcriptome-based rule set generation. Arthritis Res Ther. 2014;16:R84.

16. Tanaka T, Narazaki M, Kishimoto T. IL-6 in inflammation, immunity, and disease. Cold Spring Harb Perspect Biol. 2014;6:a016295.

17. Santos ML, Gomes WF, Pereira DS, et al. Muscle strength, muscle balance, physical function and plasma interleukin-6 (IL-6) levels in elderly women with knee osteoarthritis (OA). Arch Gerontol Geriatr. 2011;52:322-6.

18. Stannus O, Jones G, Cicuttini F, et al. Circulating levels of IL-6 and TNF-a are associated with knee radiographic osteoarthritis and knee cartilage loss in older adults. Osteoarthritis Cartilage. 2010;18:1441-7.

19. Flannery CR, Little CB, Hughes CE, Curtis CL, Caterson B, Jones SA. IL-6 and its soluble receptor augment aggrecanase-mediated proteoglycan catabolism in articular cartilage. Matrix biology: 
journal of the International Society for Matrix Biology. 2000;19:549-53.

20. Alonso N, Wani S, Rose L, Van't Hof RJ, Ralston SH, Albagha OME. Insertion mutation in Tnfrsf11a causes a Paget's disease-like phenotype in heterozygous mice and osteopetrosis in homozygous mice. Journal of bone and mineral research: the official journal of the American Society for Bone and Mineral Research2021.

21. Geerts D, Chopra C, Connelly L. Osteoprotegerin: Relationship to Breast Cancer Risk and Prognosis. Front Oncol. 2020;10:462.

22. Sparks AB, Peterson SN, Bell C, et al. Mutation screening of the TNFRSF11A gene encoding receptor activator of NF kappa B (RANK) in familial and sporadic Paget's disease of bone and osteosarcoma. Calcif Tissue Int. 2001;68:151-5.

23. Yu Z, Wang L, Li X. MiR-3150b-3p inhibits the proliferation and invasion of cervical cancer cells by targeting TNFRSF11a. J Investig Med. 2020;68:1166-70.

24. Garcia S, Hartkamp LM, Malvar-Fernandez B, et al. Colony-stimulating factor (CSF) 1 receptor blockade reduces inflammation in human and murine models of rheumatoid arthritis. Arthritis Res Ther. 2016;18:75.

25. Denny WA, Flanagan JU. Small-molecule CSF1R kinase inhibitors; review of patents 2015-present. Expert Opin Ther Pat. 2021;31:107-17.

26. Mun SH, Park PSU, Park-Min KH. The M-CSF receptor in osteoclasts and beyond. Exp Mol Med. 2020;52:1239-54.

\section{Figures}




\section{DEGs 1 \\ DEGs 2}

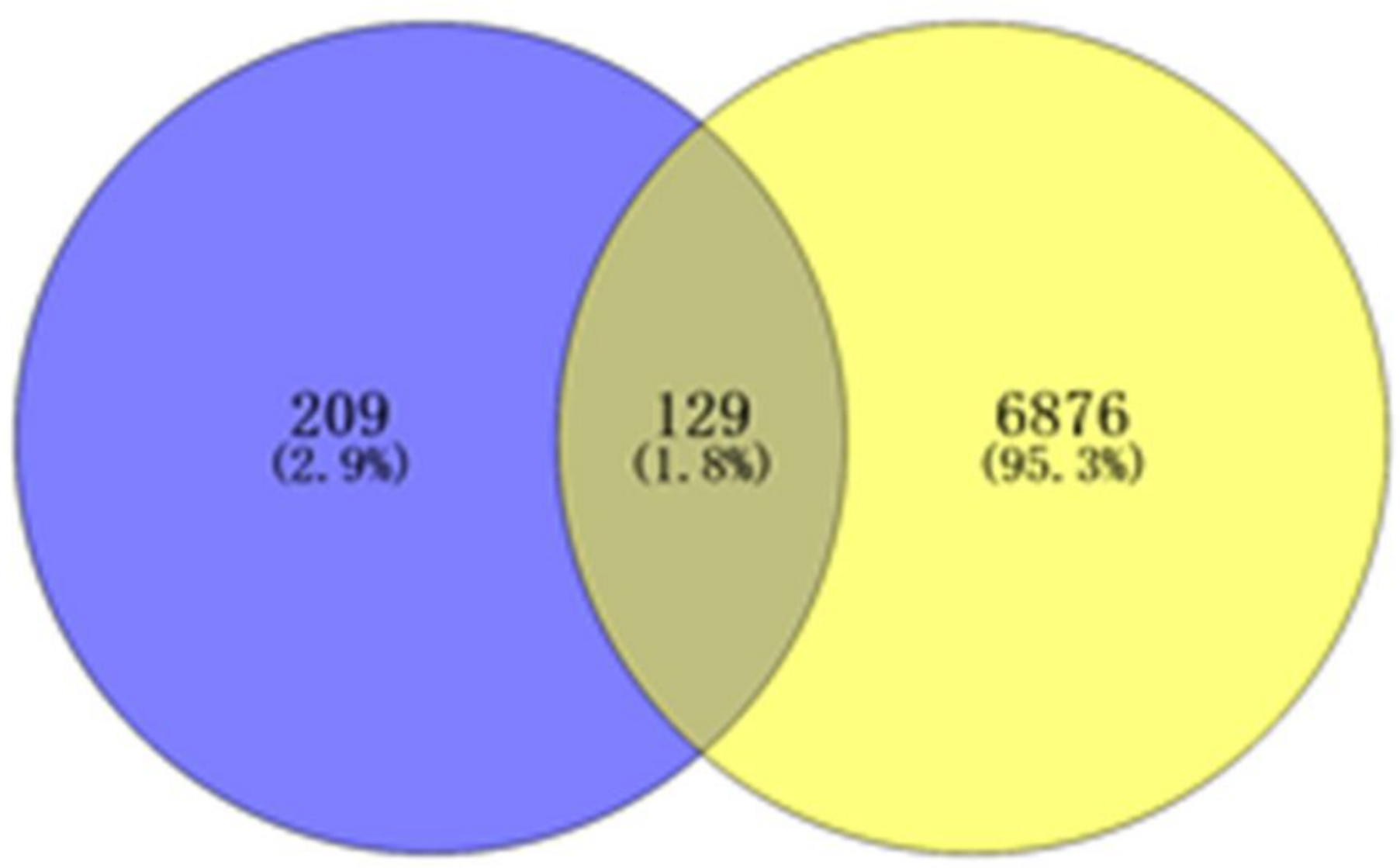

Figure 1

Screening for common DEGs from main analysis and cooperative analysis DEGs 1 for main analysis; DEGs 2 for cooperative analysis. 


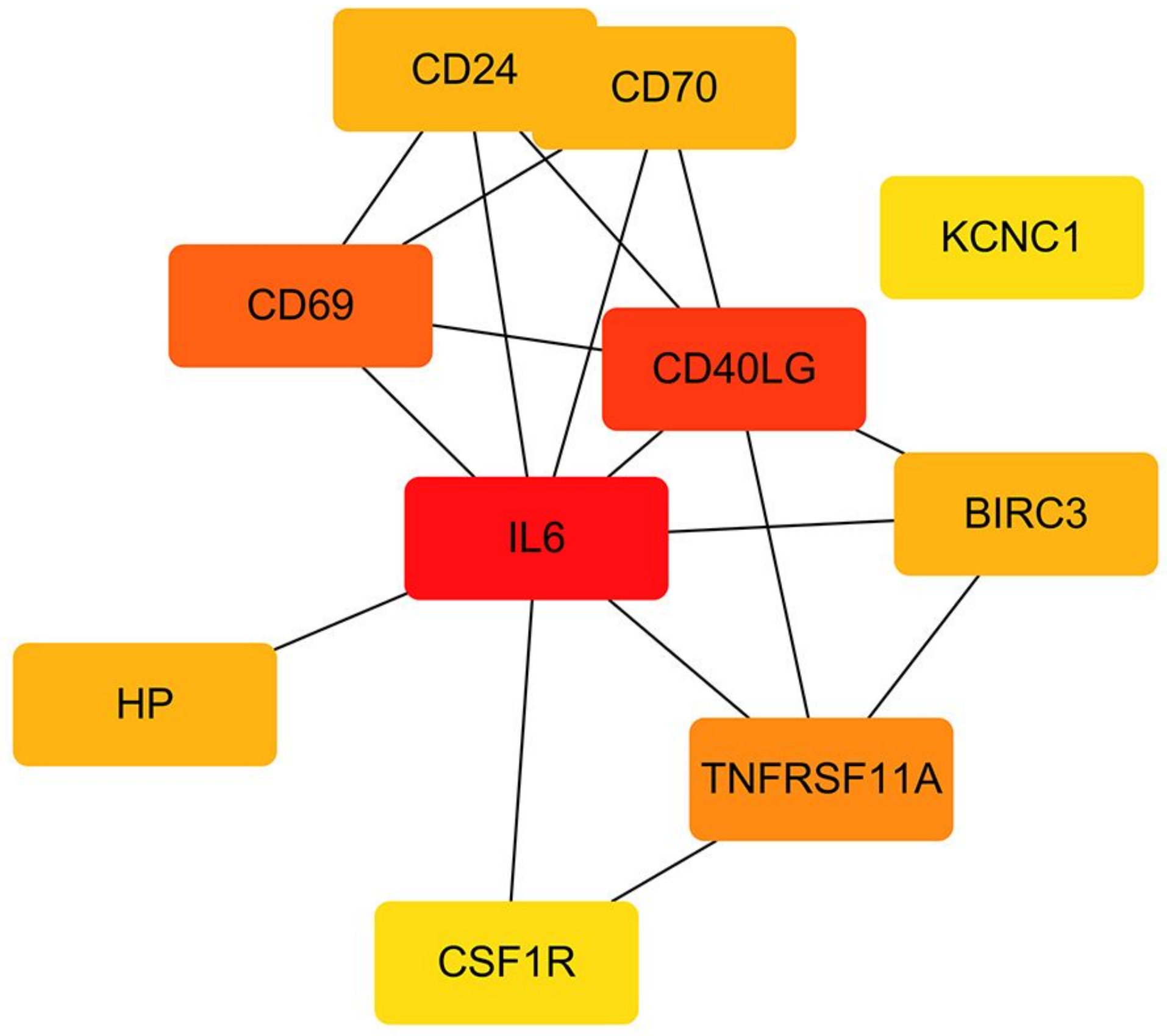

Figure 2

The top 10 hub nodes from common DEGs CD24: cluster of differentiation 24; CD70: cluster of differentiation 70; KCNC1: potassium voltage-gated channel subfamily $\mathrm{C}$ member 1; BIRC3: baculoviral IAP repeat containing 3; TNFRSF11A: tumor necrosis factor receptor superfamily member 11a; CSF1R: olony stimulating factor 1 receptor; HP: haptoglobin; CD69: cluster of differentiation 69; CD40LG: cluster of differentiation 40 ligand; IL6: interleukin 6. 